\title{
An Open Source / Freeware Assistive Technology Software Inventory
}

\author{
Alexandros Pino ${ }^{1}$, Georgios Kouroupetroglou ${ }^{2}$, Hernisa Kacorri ${ }^{1}$, \\ Anna Sarantidou ${ }^{2}$, and Dimitris Spiliotopoulos ${ }^{2}$ \\ ${ }^{1}$ National and Kapodistrian University of Athens, Accessibility Unit, \\ Panepistimiopolis, Ilissia, 15784, Athens, Greece \\ ${ }^{2}$ National and Kapodistrian University of Athens, \\ Dep. of Informatics and Telecommunications, \\ Panepistimiopolis, Ilissia, 15784, Athens, Greece \\ \{pino, koupe, c.katsori, ea05538, dspiliot\}@di.uoa.gr
}

\begin{abstract}
Assistive Technology (AT) software market is expensive, and related products are hard to find, especially for non-English speaking users. Open Source and free AT software partially solve the cost problem, and online inventories facilitate the search for the appropriate product. Even so, users don't have all the information gathered and systematically organized in one place. Furthermore, free software often needs to be tested and reviewed by computer and AT experts in order to detect and point out reliability, installation, and compatibility issues. We propose a methodology for creating web-based free AT software inventories, which will make the search and selection of such products straightforward. The methodology is based on the systematic organization and consistency of the information available for each product, and its effective presentation; the goal for the users is to be able to quickly find, compare and understand the functionality and features of each product. We have applied this methodology to create the Open Source / Freeware AT software inventory http://access.uoa.gr/fs.
\end{abstract}

Keywords: Open Source, Assistive technology, Accessibility.

\section{Introduction}

One of the main problems that Assistive Technology (AT) software users face is the high cost of such products [1]. Even when cost is not an issue, the potential AT user comes across a second obstacle: the dispersion of the locations that these products can be found; information for each product is available on its own website, with no easy way to overview all available software in one place. Online free AT software inventories or lists try to address these challenges. Other problems that arise when a specific product is about to be selected include compatibility issues and the limited number of languages that it usually supports; in many cases the software is not compatible with the user's computer or operating system [2]. Moreover, for non-English speaking users, finding an AT software that "speaks" their own language is often a difficult task. Furthermore, there is no reassurance that a free AT application is tested by a 
specialist team in terms of usability and stability [3]. Free software usually does not have warranty, support or extensive documentation available, so testing it is an important issue. Finally, in existing inventories there is no consistent way of presenting AT applications with the same level of information detail and covering all important features. In order to facilitate disabled users to find, select, and afford AT, we propose a methodology for creating a well-structured, easy to use and understandable online inventory of tested, free of charge AT software products.

Free of charge software can be found in various forms: Open Source software, freeware, shareware, and trial versions of commercial software [4], [5]. Open Source ${ }^{1}$ software means that the source code, as well as the compiled form of the application is freely redistributed [6]. Freeware ${ }^{2}$ is computer software that is made available free of charge, but is copyrighted by its developer, who retains the rights to control its distribution, modify it and sell it in the future. It is typically distributed without its source code, thus preventing modification by its users. Shareware ${ }^{3}$ is software that is distributed freely or on a trial basis with the understanding that the user may need or want to pay for it later. Some software developers offer a shareware version of their application with a built-in expiration date, after which the user can no longer get access to the program. Other shareware (sometimes called liteware) is offered with certain capabilities disabled as an enticement to buy the complete version of the product. Trial versions of commercial software is similar to shareware or liteware, with the difference that shareware can sometimes be used for an unlimited period of time free of charge, while trial versions will certainly expire and need to be purchased eventually.

\section{Related Work}

Several lists of free AT software can be found on the Internet: The Open Source Assistive Technology Software (OATSoft ${ }^{4}$ ) website currently (April 2010) lists 162 Open Source projects [7]. A brief description for each project is given as well as the possibility to download the software and/or source code from an external developer's site. There are three ways to search the OATSoft inventory, namely based on the needs that the software meets (options include: Text Input, Communication, Using the Mouse, Viewing the Screen, Accessing the Web, Symbols, Alternative Access, Learning and Education, General Tools, and Other Need), by type of software (options include: On Screen Keyboards, Symbol Libraries, Text to Speech, Computer Automation, Switch Input Software, Alternative and Augmentative Communication, Environmental Control, Educational and Learning, General Tools, and Other Function), or by simply listing the whole inventory. OATSoft website also includes a rating system (scale 1-Average to 5-Brilliant) for each product and the possibility to add comments on each product's page. Project:Possibility ${ }^{5}$ website provides a smaller inventory of Open Source AT projects that currently lists 16 applications. Projects are divided into six sections, namely Educational, Intelligent Input, Mobile, Music, Video Game, and

\footnotetext{
${ }^{1}$ http://www.opensource.org/docs/definition.php

${ }^{2}$ http://www.linfo.org/freeware.html

${ }^{3}$ http://searchenterpriselinux.techtarget.com/sDefinition/0,sid39_gci212977,00.html

${ }^{4}$ http://www.oatsoft.org/

${ }^{5}$ www.projectpossibility.org/
} 
Web. Raising the Floor $^{6}$ website lists 13 Open Source projects divided into three sections: a) for individuals with blindness, low-vision or reading difficulties, b) for individuals looking for assistance with reading skills, and c) for web content developers. Other websites listing a number of Open Source, freeware, and shareware AT applications, and offering the corresponding files for download, include Emptech ${ }^{7}$ (134 applications), and Adaptech ${ }^{8}$ (124 applications).

All of the inventories mentioned above list AT software applications without taking into account or commenting on features like usability, stability, and functionality. This would require testing of each application by AT specialists, and the inclusion of results/comments about, for example, the installation process, the settings options, and the actual language support of each individual AT application [8]. The existing aforementioned websites do not include such data.

Among the most important characteristics of our proposed methodology for building an AT inventory that set this work apart from what already exists in the Internet is that all the included applications have been already installed and tested by experts (authors of the AT software inventory). That means that there is a high degree of reassurance for the users that all software included in the list works as intended [9]. This is quite helpful especially for novice or non expert users that don't have the time, capability or knowledge to test or evaluate the software themselves. Especially for users from countries with not common or widespread languages, like Greek, testing each piece of software for local language support is quite important.

\section{Methodology}

The proposed methodology consists of seven steps described in the next sections. Each section contains details of our implementation as a proof of concept for good practice.

\subsection{Searching and Locating Free AT Software Available on the Internet}

Resources explored include all the inventories mentioned in section 2, as well as links found in user forums and additional AT vendors' websites for individual applications. A total number of 224 individual applications were found. Among these, 46 of the applications, although they were characterized as AT software in an inventory, they were actually general purpose software or irrelevant to any disability, so they were excluded from our list (for example office automation, web browsing or gaming software without any special accessibility options or features).

\subsection{Installing the Application}

All applications were installed on computers with the Operating Systems they supported. A total number of 19 applications failed to successfully complete the installation process; in most of them, either installation files were missing or purchase was needed in contrast of what their description stated.

\footnotetext{
${ }^{6} \mathrm{http}: / /$ raisingthefloor.net/

${ }^{7}$ http://www.emptech.info/categories_list.php

${ }^{8}$ http://adaptech.dawsoncollege.qc.ca/fandi_e.php
} 


\subsection{Testing the Application}

Subsequent to installation, all the applications were launched and tested, in order to investigate whether they realize the functionality their documentation or description depicted. Program stability was also tested for 3 hours of operation for each application. Different settings and layouts were tested where applicable. A total of 14 applications failed to run smoothly, exhibiting stops and crashes at run-time, so they were excluded. As our inventory has to address a disabled community speaking a less common language, all software that would be unusable by Greek users was excluded, for example, text to speech software that does not "speak" Greek, or screen readers that do not recognize Greek fonts. 63 applications were evaluated as unusable for Greek users specifically. At the end of all filtering and testing, 82 free AT software applications were selected to populate our inventory. Table 1 summarizes the number of applications processed in the first three steps.

Table 1. AT software located, tested, and selected during the application of our methodology

\begin{tabular}{lrr}
\hline & number & percentage \\
\hline Total applications located & 224 & $100 \%$ \\
Non-AT applications (excluded) & 46 & $21 \%$ \\
Applications failed to install (excluded) & 19 & $8 \%$ \\
Applications failed to run (excluded) & 14 & $6 \%$ \\
Applications not supporting Greek language (excluded) & 63 & $28 \%$ \\
Applications finally selected for our inventory & 82 & $37 \%$ \\
\hline
\end{tabular}

\subsection{Documenting}

After the successful testing of each free AT software, the application was documented and the following fields of information were filled:

- Application name: The name of the free AT application.

- Developer: The company's or individual developer's/author's name, with a link to the corresponding website where available.

- Version: The version number of the application along with the characterization "open source" or "freeware" or "shareware".

- Related category(ies): All applications were classified in one or more of the following categories: Voice recognition, Screen reader, Daisy, Calculator, Mouse pointers, Click helper, Virtual keyboard, Camera mouse, Alternative communication, Text to speech, Screen magnifier, Braille translator, Web browser, Mouse emulator, Contrast adjustment, Keyboard shortcuts, Voice mail, Clock, Video call. Categories can be added when free software of a new category appears.

- Related disability(ies): All applications were also classified according to one or more disabilities: Speech, Hearing, Motor, Blindness, Low Vision. Additional disabilities can be added.

- Description: A description of the functionality of each application. The most important features of the AT application are listed in this field. Special or unique features that set each application apart from its competitors are stressed out. 
- System requirements: The Operating Systems on which the free application runs were listed, and machine requirements like free hard disk space, RAM memory, screen resolution, etc.

- Installation notes: The software installation procedure is explained in this field, starting with which file the user has to run first, and focusing on tricky steps of the installation procedure when such steps exist (for example, if further prerequisites are required for the program to run, how to set it up on a browser in the case it is a browser add-on, how to unzip the installation files when needed, etc.).

- Settings: An important aspect of the documentation that is not present in any other online inventory. The main settings of the AT software are analyzed and hints are given to the potential users about the best way to setup the program according to their needs. Language settings were included, as well as comments on what effect each available option has to the operation of the software. This way, users can always know whether the application has the adaptation features they're searching for without having to download and install all available software and explore the settings menus by themselves, often making several "trial and error" circles.

- Internal download link: All software is gathered in our inventory's file server, offering a local link for fast download for the potential users. This way we avoid the possibility of unavailable files due to remote server downtimes, disappeared websites, or discontinued projects.

- External download link: The original external download link is also given.

- Screenshot(s): A typical screenshot image of the running application.

- Comments: The text in this field is written by the members of our research team who tested the software, and includes opinions like how easy is to use the application, tips about its operation, etc.

- Help: Information about the existence of a help system built-in the application, availability of user manual, and in some cases tutorials (built-in or online).

- Language: Supported languages in the applications menus, help files, user manuals, text-to-speech, Unicode support, with emphasis to local language.

- License: The type of the user license of each application is given when available.

- Product home page: A link to the original product home page is given here.

- Additional links: Additional links include web pages with reviews of the product, previous versions, current issues or bugs, future plans of the developers, etc.

- Related software: This field provides links to other software in our database that is similar to the current software, or to dependent software (for example, a web browser will be linked to a browser add-on's page).

- File size: The size of the file that the user has to download in order to install or run the application.

\subsection{Usable Online Inventory Design}

The new web-based inventory for Open Source, freeware and non-expiring shareware AT software was designed having usability and ease of use in mind. There are three ways to search for a free AT application: a) by disability - selecting one of the related disabilities mentioned above, b) by application category - selecting one of the related categories, and c) selecting to view all available applications in an alphabetical order. 
This 3-way navigation menu is available at all web pages of the inventory. Each individual application is described in its own web page that contains all information listed in the Documenting step, as well as user rating results, user comments, and statistics showing numbers of visitors and downloads. Thus, each AT software application's web page is enriched with user evaluation data (1 to 5 star rating by users) as well as comments, tips and tricks, or complaints that users make on the comments section. Illustrative disability category icons are always next to each application's name in order to depict related disabilities.

\subsection{Accessible Website Development}

The website http://access.uoa.gr/fs was built according to W3C Web Content Accessibility Guidelines $1.0^{9}$, and a Level Triple-A Conformance was achieved. The CSS level 2.1 Conformance was reached (based on the W3C CSS Validation Service), as well as the HTML 4.01 Transitional compatibility (based on W3C's Markup Validation Service) [10].

\subsection{Update and Maintenance}

Our research team members are often checking for new versions of all free AT software of the inventory. Every time a new version is available, the old one is replaced and all documentation information is updated, while old versions history is kept. The date and time of the last modification that was done to an application's information fields is noted, and a "recent changes" section informs visitors about all additions, deletions and modifications made. We have also subscribed to several mailing lists, RSS feeds and user forums, as additional sources of information for new releases, as well as newly created AT applications that need to be tested and added. Finally, user and visitor contributions like comments on individual applications' pages, problem reports, and e-mails are often very useful, as they recommend new software to be added, or make comments on usability issues for the already included applications, as well as their own experience on using free AT software.

\section{Discussion}

Table 2 summarizes the information given explicitly for each product (in terms of fields described in 3.4), and the most important features of the corresponding inventory websites. It is made clear that the inventory we propose provides systematically (in individual fields and not in a general description text) all necessary information and details needed for each AT software application it contains.

The free AT software inventory http://access.uoa.gr/fs has been online for six months at the time that this paper was written. This work has been disseminated through the press, special interest mailing lists and announcements to all disability organizations and associations. The first reactions and feedback of the Greek disabled community and related professionals are positive. The website statistics show 94,528 hits and 1,886

${ }^{9}$ http://www.w3.org/TR/WCAG10/ 
Table 2. Overview of the information fields given for each product, and the most important website features for A: OATSoft, B: Project:Possibility, C: Raising the Floor, D: EmpTech, E: Adapttech, and F: http://access.uoa.gr/fs.

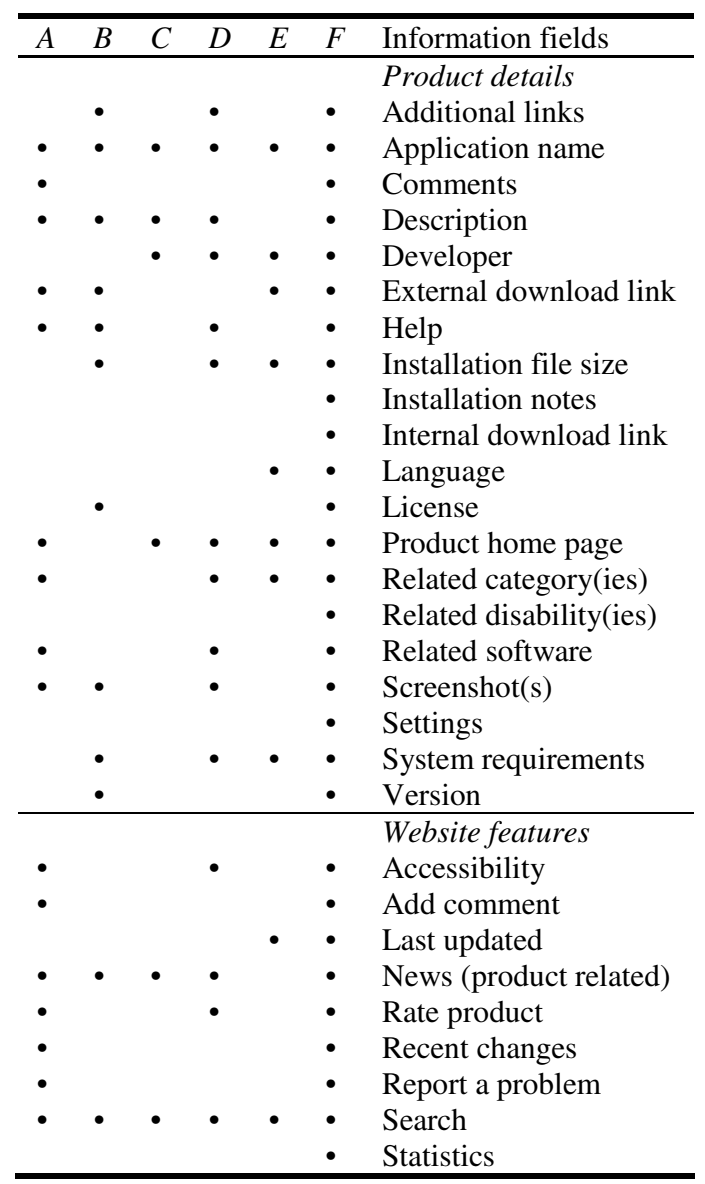

downloads by 13,440 unique visitors. $78 \%$ of the visitors were from Greece and the rest accessed the web site from abroad. We have also received more than 150 user messages (comments on products, product rating entries, and e-mails).

\section{Future Work}

Future plans include the addition of a user forum about AT software, as well as a "most popular applications" section, according to downloads statistics that are collected. The inventory will be continuously updated and new software will be added; we are especially looking forward to the free AT software contributions by the ongoing AEGIS [11] and Accessible ${ }^{10}$ European projects.

${ }^{10} \mathrm{http}: / /$ www.accessible-eu.org/ 
Acknowledgments. The work described in this paper has been funded by the Special Account for Research Grants of the National and Kapodistrian University of Athens.

\section{References}

1. Vanderheiden, G.C.: Redefining Assistive Technology, Accessibility and Disability Based on Recent Technical Advances. J. Technology in Human Services 25, 147-158 (2007)

2. Emiliani, P.L.: Assistive Technology (AT) Versus Mainstream Technology (MST): The Research Perspective. Technology and Disability 18, 19-29 (2006)

3. Law, C.M., Yi, J.S., Choi, Y.S., Jacko, J.A.: Are Disability Access Guidelines Designed for Designers?: Do They Need to Be? In: 18th Australia Conference on Computer-Human Interaction: Design: Activities, Artifacts and Environments, pp. 357-360. ACM Press, New York (2006)

4. Chopra, S., Dexter, S.: Decoding Liberation: A Philosophical Investigation of Free Software. Routledge, New York (2007)

5. Morelli, R., Tucker, A., Danner, N., De Lanerolle, T.R., Ellis, H.J., Izmirli, O., Krizanc, D., Parker, G.: Revitalizing Computing Education Through Free and Open Source Software for Humanity. Communications of the ACM 52, 67-75 (2009)

6. Richle, D.: The Economic Motivation of Open Source Software: Stakeholder Perspectives. IEEE Computer 40, 25-32 (2007)

7. Judge, S., Lysley, A., Walsh, J., Judson, A., Druce, S.: OATS - Open source assistive technology software - a way forward. In: 12th Biennial Conference of the International Society for Augmentative and Alternative Communication, ISAAC (2006)

8. Savidis, A., Stephanidis, C.: Inclusive Development: Software Engineering Requirements for Universally Accessible Interactions. Interacting with Computers 18, 71-116 (2006)

9. Crowston, K., Howison, J., Annabi, H.: Information Systems Success in Free and Open Source Software Development: Theory and Measures. Softw. Process Improve. Pract. 11, 123-148 (2006)

10. Votis, K., Lopes, R., Tzovaras, D., Carriço, L., Likothanassis, S.: A Semantic Accessibility Assessment Environment for Design and Development for the Web. In: Stephanidis, C. (ed.) HCI 2009. LNCS, vol. 5616, pp. 803-813. Springer, Heidelberg (2009)

11. Korn, P., Bekiaris, E., Gemou, M.: Towards Open Access Accessibility Everywhere: the AEGIS Concept. In: Stephanidis, C. (ed.) HCI 2009. LNCS, vol. 5614, pp. 535-543. Springer, Heidelberg (2009) 